\title{
Factores e intervenciones que inciden en las condiciones y medio ambiente de trabajo para incrementar la atracción, captación y retención de recursos humanos en salud en el primer nivel de atención de áreas rurales, remotas o desatendidas
}

\author{
María Eugenia Esandi 1, Laura Antonietti' , Zulma Ortiz', Malhi Cho², Isabel Duré3, Ludovic Reveiz² \\ y Fernando Menezes ${ }^{2}$
}

Forma de citar

Esandi ME, Antonietti L, Ortiz Z, Cho M, Duré I, Reveiz L et al. Factores e intervenciones que inciden en las condiciones y medio ambiente de trabajo para incrementar la atracción, captación y retención de recursos humanos en salud en el primer nivel de atención de áreas rurales, remotas o desatendidas. Rev Panam Salud Publica. 2020;44:e112. https://doi.org/ 10.26633/RPSP.2020.112

RESUMEN

Objetivo. Identificar y sistematizar la evidencia empírica disponible sobre factores e intervenciones que inciden en las condiciones y medio ambiente de trabajo para incrementar la atracción, captación y retención de recursos humanos en salud en el primer nivel de atención de áreas rurales, remotas o desatendidas.

Métodos. Revisión rápida de revisiones, seleccionadas según criterios de pertinencia, elegibilidad e inclusión con búsquedas en bases de datos electrónicas, de literatura gris y manual. Se empleó el AMSTAR I para evaluar la calidad de revisiones sistemáticas y un análisis temático para síntesis de resultados.

Resultados. Se incluyeron 16 revisiones, una de las cuales contenía a su vez 14 revisiones. Del total, 20 revisiones analizaron factores y 9 evaluaron efectividad de intervenciones. La evidencia sobre factores es abundante, pero de limitada calidad. Los factores individuales, familiares y la "exposición rural previa" se asociaron a mayor captación; factores organizacionales y del contexto externo fueron gravitantes en la retención. El trabajo en red y el apoyo profesional incidieron en la captación y retención. La evidencia sobre efectividad de intervenciones fue limitada, en cantidad y calidad. El tipo de intervención más frecuentemente empleada fueron los incentivos.

Conclusiones. La evidencia sobre factores que se relacionan positivamente con la captación y retención de trabajadores en el primer nivel de atención de áreas rurales, remotas o desatendidas es suficiente y debería ser tenida en cuenta al diseñar intervenciones. La evidencia de calidad sobre la efectividad de intervenciones es escasa. Se requieren más estudios controlados con rigurosidad metodológica, particularmente en las Américas.

Palabras clave Condiciones de trabajo; medio rural; zonas remotas; área sin atención médica; recursos humanos; atención primaria de la salud; selección de personal.

\footnotetext{
1 Academia Nacional de Medicina, Buenos Aires, Argentina $\square$ María Eugenia Esandi, eesandi@gmail.com

2 Organización Panamericana de la Salud, Washington, DC, Estados Unidos de América
} 
Avanzar hacia el logro de los Objetivos de Desarrollo Sostenible requiere disponer de recursos humanos en salud (RHS) en número suficiente y con perfiles adecuados y calificados para atender efectivamente las necesidades de la población; particularmente en servicios de atención primaria de salud (1). Todos los países enfrentan brechas de cobertura sanitaria y acceso a servicios de salud entre poblaciones rurales y urbanas, pero en aquellos de bajos y medianos ingresos el problema es aún más acuciante.

Resolver las desigualdades en la distribución de los RHS, entendidos como todo personal que realiza tareas que tienen como principal finalidad promover la salud, es sumamente difícil por diversas razones:

i) la complejidad inherente al problema, en el cual intervienen diferentes factores que operan desde la etapa de formación hasta la captación o retención de los RHS; las políticas públicas, en particular aquellas referidas a RHS (2); la organización del sistema de salud y las características del individuo y su familia.

ii) Frente a la existencia de múltiples enfoques y marcos conceptuales para el análisis de las condiciones y medio ambientes de trabajo (CyMAT) es posible que la selección de intervenciones no sea pertinente para el ámbito de aplicación.

iii) La limitada evidencia sobre la implementación y efectividad de las políticas, planes y programas destinados a mejorar la atracción, captación y retención de RHS, en especial, en América Latina y el Caribe $(2,3)$.

Con el propósito de contribuir a identificar las brechas de conocimiento que limitan la toma de decisiones destinadas a mejorar la disponibilidad de RHS en el primer nivel de atención de áreas remotas, rurales y desatendidas (RRD) se revisó la literatura considerando cuatro categorías de intervenciones: educación, regulación, incentivos financieros, y apoyo, tanto profesional como personal (4). Para la definición de las CyMAT se empleó un modelo conceptual ad hoc, que incluye factores del individuo y su entorno cercano; factores organizacionales, condiciones de empleo y trabajo y factores del contexto externo (5).

El objetivo de la revisión fue identificar y sistematizar la evidencia disponible proveniente de revisiones sobre factores e intervenciones que inciden en las CyMAT para incrementar la atracción, captación y retención de RHS en el primer nivel de atención de áreas RRD.

\section{MATERIALES Y MÉTODOS}

Se realizó una revisión rápida de la literatura mediante la búsqueda sistemática de estudios secundarios y terciarios publicados en bases de datos electrónicas (PubMed, Lilacs); metabuscadores (Epistemonikos); biblioteca de la Colaboración Cochrane; literatura gris en Google Académico; búsqueda manual por referencias de artículos incluidos y consultas a informantes clave.

La búsqueda se realizó por palabras clave y se organizó en cuatro bloques temáticos definidos a partir de los componentes de la pregunta de investigación: ¿qué factores e intervenciones inciden en las CyMAT (bloque 1) en el Primer Nivel de Atención (bloque 2) en áreas RRD (bloque 3) y se asocian o tienen efecto en: a) la atracción, la captación o la retención de los trabajadores de la salud; b) la mejora de la salud, motivación, satisfacción y desempeño de los trabajadores de la salud? (bloque 4). Se emplearon múltiples fuentes de información y filtros por tipo de estudio ("revisión sistemática") y año de publicación (entre 2014 y 2019) (la estrategia completa está disponible previa solicitud a los autores).

\section{Selección}

Se tuvieron en cuenta criterios de pertinencia (revisiones cuyo título incluyera palabras relacionadas con los bloques 1 y 4), de elegibilidad (artículos pertinentes cuyo título o resumen incluyera palabras relacionadas con todos los bloques temáticos) y de inclusión. Se definieron criterios de inclusión según tipo de estudio -terciarios como revisión de revisiones y secundarios, como revisiones sistemáticas (RS) y otros tipos de revisiones, en idioma inglés, portugués y español-; tipo de intervención -según la clasificación propuesta por la OMS (4) o factores que inciden en CyMAT-; tipo de participantes -trabajadores/ as de la salud, profesionales o no profesionales, de distintas disciplinas que se desempeñaran en el primer nivel de atención-; ámbito geográfico -áreas RRD-; y desenlace de interés primarios: atracción, captación o retención; secundarios: bienestar, motivación, satisfacción y desempeño profesional-.

Se excluyeron estudios primarios y revisiones narrativas por su mayor riesgo de sesgo en comparación con otros tipos de revisiones que emplean métodos sistemáticos de búsqueda y análisis de resultados; estudios sobre intervenciones dirigidas a estudiantes o sobre personal que se desempeña en el segundo o tercer nivel de atención; estudios que no incluyeran evidencia sobre áreas RRD; estudios que abordaran medidas de desempeño a nivel del sistema o del usuario final y aquellos que no consideraran al menos uno de los desenlaces primarios (atracción, captación o retención).

La selección se realizó de manera secuencial en tres fases: 1) selección de artículos pertinentes por una revisora a partir de la lectura del título de la publicación (MEE); 2) selección de artículos elegibles por dos revisoras a partir de la lectura del título y resumen (ZO; LA); aquellos calificados como "no elegibles" fueron evaluados por una tercera revisora (MEE), con resolución de las discrepancias por consenso; 3) selección de artículos incluidos por dos revisoras de manera independiente a partir de la lectura del texto completo (LA; MEE) con resolución de las discrepancias por una tercera revisora (ZO). Se evaluó la calidad de las RS incluidas por medio del AMSTAR I.

\section{Extracción y síntesis de los datos}

Fue realizada por dos revisoras (MEE; LA) e incluyó datos generales de la revisión (año de publicación, primer autor, objetivo, participantes, ámbito geográfico, países y estrategia de búsqueda empleada); tipo de intervención según clasificación de la OMS (4); calidad de los estudios; descripción de resultados: a) sobre factores: se identificaron aquellos que mostraron una asociación positiva o negativa con los desenlaces primarios (atracción, captación y retención) y se sintetizaron los hallazgos sobre asociaciones positivas en una matriz comparativa de las RS incluidas; b) Sobre intervenciones: efecto reportado en los estudios incluidos en la revisión (positivo o 
negativo), independientemente del indicador empleado en cada caso.

\section{RESULTADOS}

Se incluyeron en total 29 revisiones, 20 sobre factores y 9 sobre intervenciones (figura 1).
Evidencia sobre factores que inciden en la atracción, captación y retención de trabajadores en áreas rurales, remotas y desatendidas

Se identificó una revisión de RS (6-19) publicada por Mbemba y cols. en el año 2015 (20) sobre factores que influencian la captación y retención de trabajadores de salud en áreas rurales y

FIGURA 1. Flujograma de selección de artículos

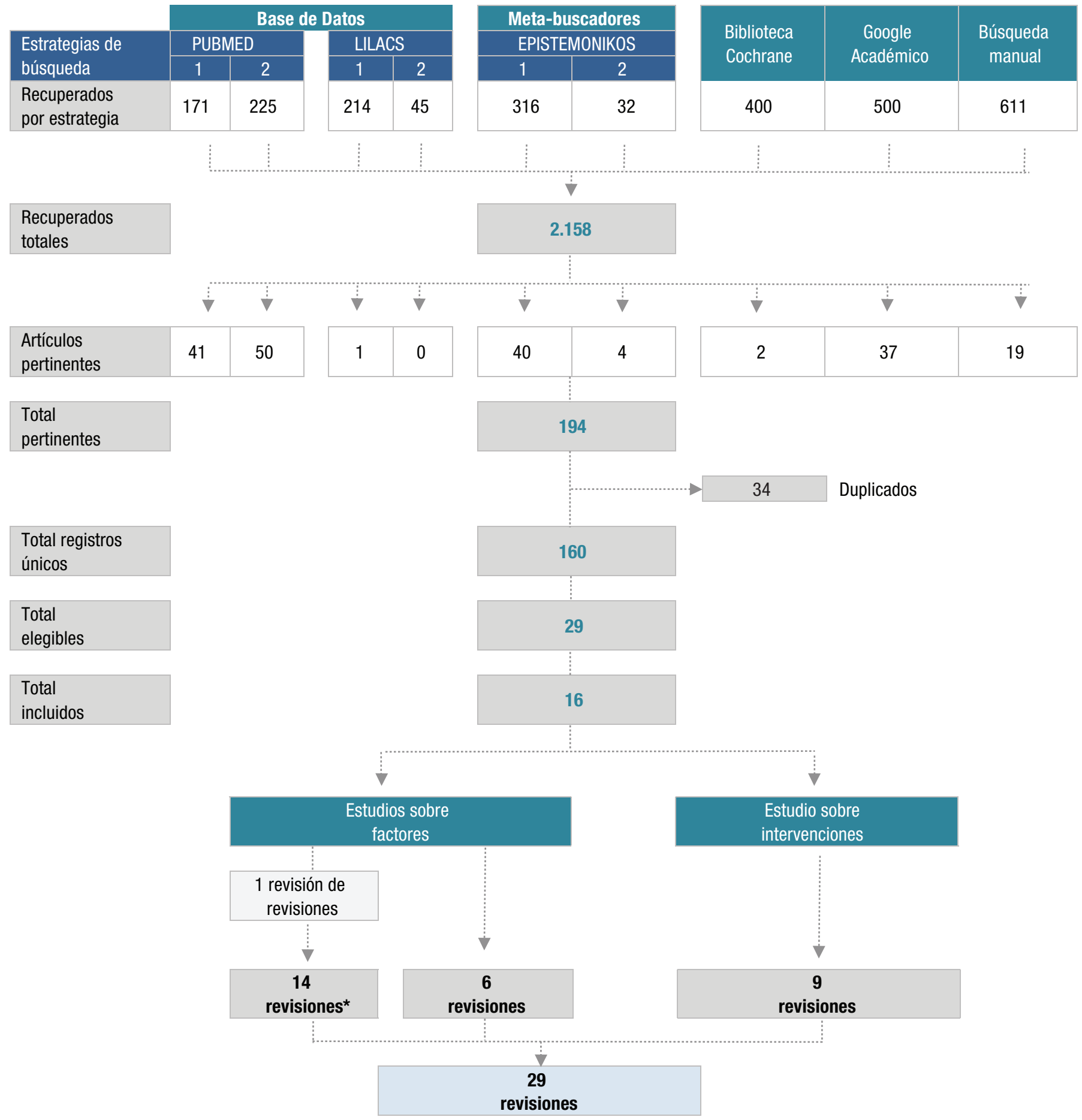

Fuente: elaboración propia a partir de datos de la revisión.

*Se excluye una revisión por tener una población objetiva diferente (estudiantes de ciencias auxiliares) 
CUADRO 1. Principales características de las revisiones que estudiaron factores asociados a la captación y retención de personal de salud en áreas remotas, rurales o desatendidas

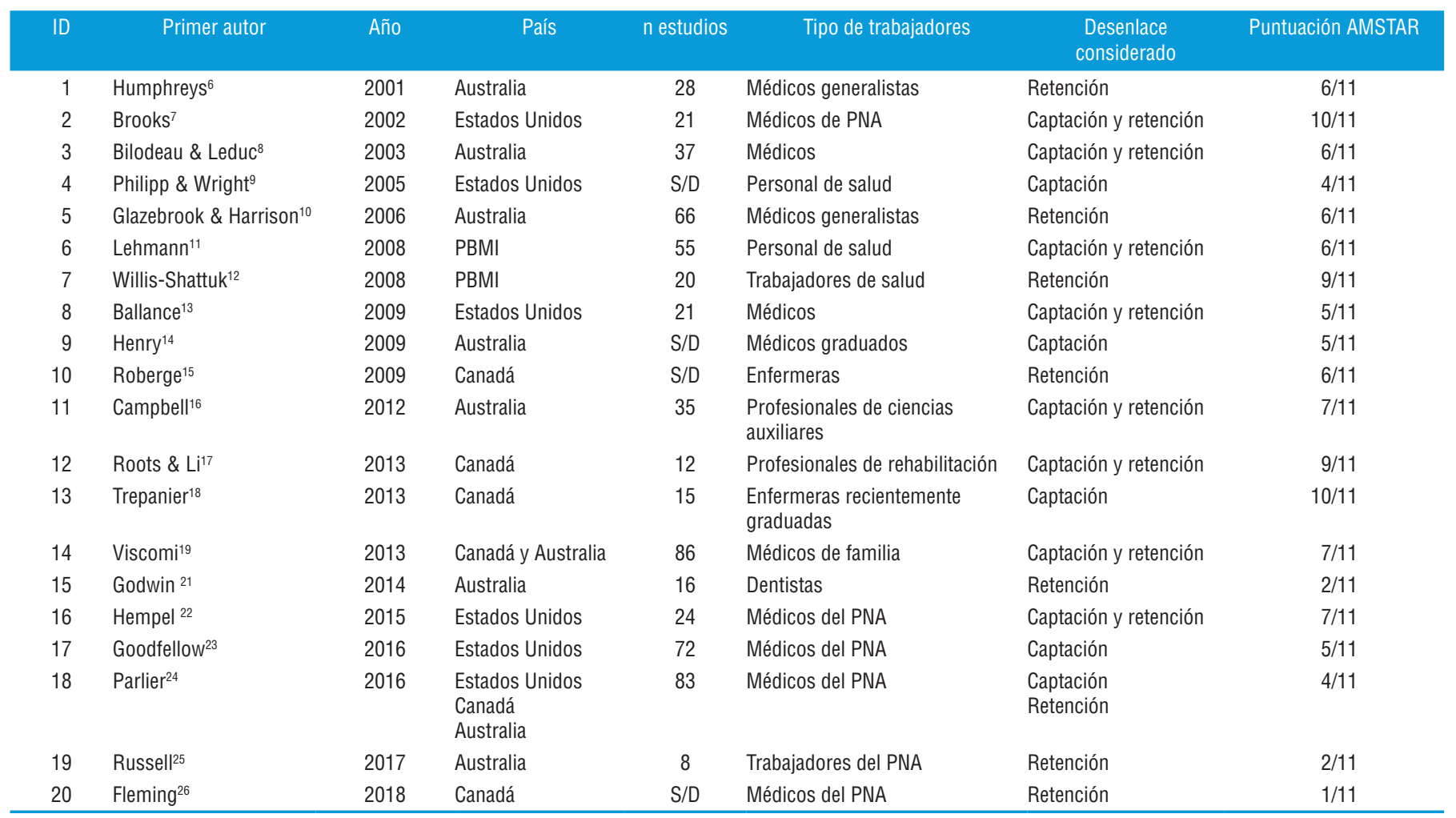

Fuente: elaboración propia a partir de datos de la revisión y datos tomados del cuadro 2 de Mbemba y cols (20) PNA, primer nivel de atención

remotas, y seis revisiones posteriores (21-26). En total, se analizaron 20 RS: 14 identificadas en la revisión de Mbemba -una se excluyó por tratarse de estudiantes- y 6 RS identificadas a través de la revisión rápida (cuadro 1).

El rango de estudios incluidos en cada una de las revisiones varió entre 8 (25) y 86 (19). La calidad de las 6 RS publicadas posteriormente a la publicación de Mbemba (20) fue muy baja, a excepción de la de Hempel (22). La media de ítems del AMSTAR alcanzados en las RS identificadas por Mbemba y cols. fue de 5,8 mientras que en las RS más recientes fue sólo 3,5.

Sobre la base del modelo conceptual propuesto y los resultados de las revisiones, se identificaron siete categorías de factores asociados a la captación, la retención o ambos desenlaces (cuadro 2).

1) Factores individuales: quince RS señalaron el "origen rural" o la crianza en ámbitos rurales como uno de los predictores más fuertes de la captación y retención de RHS en áreas RRD (7-9,13,14,17-19,21-24,26). Dos revisiones realizadas en Estados Unidos identificaron la pertenencia o dominio del idioma de una minoría como un factor asociado a la decisión de trabajar en áreas desatendidas donde residen esos grupos, predominantemente asiáticos o latinos $(22,23)$. No se encontraron asociaciones significativas con género y edad, a excepción de una RS que identificó mayor predisposición del género masculino a elegir la práctica rural como destino (24), y una RS que señaló mayor retención entre trabajadores en la etapa media de su vida comparados con los más jóvenes o ancianos (25).
2) Factores familiares: cinco RS jerarquizaron las oportunidades laborales de la pareja o las facilidades para acceder a la educación de sus hijos/as como factores que inciden en la captación $(23,24)$ y en la retención de trabajadores/as en áreas $\operatorname{RRD}(10,23,25,26)$.

3) Factores vinculados con la exposición o entrenamiento durante la formación de grado: además del origen o la crianza rural, la "identidad rural" puede lograrse a través de exposiciones repetidas a estos ámbitos, que permiten conocer la cultura, las formas en las que las personas se relacionan, y las características de la práctica rural (24). Tanto el antecedente de una formación de grado orientada a especialidades rurales como las rotaciones rurales fueron identificadas como favorecedoras de la captación de profesionales en estos ámbitos (7-9,13,14,18,19,21-24). Sin embargo, es importante garantizar que los participantes sean adecuadamente preparados para abordar las complejidades de la práctica en estos entornos y que reciban soporte apropiado durante la rotación (24).

4) Factores vinculados con la exposición o entrenamiento en la formación de posgrado: tanto la realización de una residencia en especialidades vinculadas a la APS $(23,24,26)$ como una rotación en ámbitos rurales se asociaron positivamente con la captación $(7-9,14,18,19,22,23)$ y en menor medida, con la retención de RHS en ámbitos rurales (21).

5) Factores financieros: incluyen la deuda asumida durante el período de formación a cambio de servicios postgraduación en determinados ámbitos, e incentivos financieros y el salario, que se asociaron a mejoras en la captación y retención de RHS en 8 revisiones $(9-12,18,23,24,25)$. 
CUADRO 2. Factores asociados positivamente con cada tipo de desenlace considerado en las revisiones identificadas

\begin{tabular}{ccccccccccccccccccccccccccc}
\hline Desenlace considerado & C & C & C & C & C & R & R & R & R & R & R & R & R & CR & CR & CR & CR & CR & CR & CR & CR \\
\hline ID RS & 4 & 9 & 13 & 17 & 18 & 1 & 5 & 7 & 10 & 15 & 18 & 19 & 20 & 2 & 3 & 6 & 8 & 11 & 12 & 14 & 16
\end{tabular}

Factores individuales

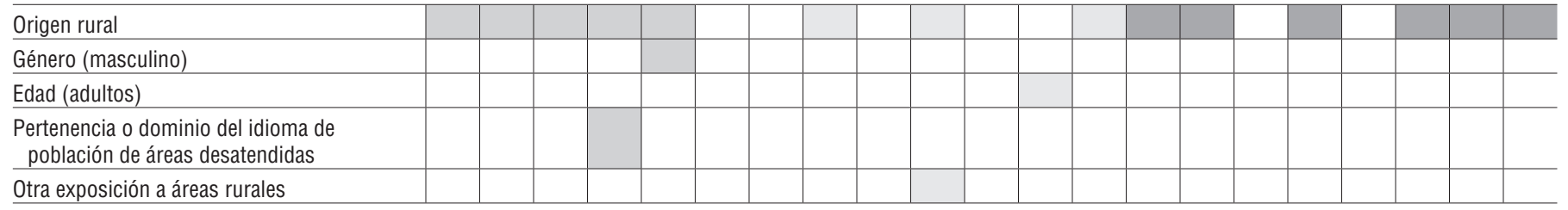

\section{Factores familiares}

Satisfacción de preferencias o necesidades de

la pareja o hijos/as

Exposición o entrenamiento en la formación de grado

Currícula con contenidos de medicina familiar o rural

Rotación clínica en área rural

Exposición o entrenamiento en la formación de posgrado

Residencia en Medicina Familiar o APS

Rotación clínica en área rural

Factores financieros \begin{tabular}{l|l|l|l|l|l|l|l|l|l|l|l|l|l|l|l|l|l|l|l|l|l}
\hline $\begin{array}{l}\text { Participación en programas de reembolso de } \\
\text { deuda educacional }\end{array}$ & & & & & & & & & & & & & & & & & & & & \\
\hline Incentivos financieros y salario & & & & & & & & & & & & & & & & & & \\
\hline
\end{tabular}

Características de la práctica rural y condiciones de trabajo

\begin{tabular}{l|l|l|l|l|l|l|l|l|l|l|l|l|l|l|l|l|l|l|l|l|l}
\hline Características de la práctica rural & & & & & & & & & & & & & & & & & & & & & \\
\hline Trabajo en red y apoyo profesional & & & & & & & & & & & & & & & & & & & & \\
\hline $\begin{array}{l}\text { Condiciones de trabajo } \\
\text { Oportunidades de desarrollo de la carrera } \\
\text { profesional }\end{array}$ & & & & & & & & & & & & & & & & & & & & \\
\hline Educación médica & & & & & & & & & & & & & & & & & & & & & \\
\hline Autonomía
\end{tabular}

Autonomía

Comunidades y estilo de vida rural

Condiciones de vida generales

Estilo de vida rural y vinculación con la comunidad

Fuente: elaboración propia a partir de resultados de la revisión.

C, Captación; R, Retención; APS, Atención Primaria de la Salud

6) Factores vinculados con las características de la práctica rural y condiciones de trabajo: el trabajo en red, apoyo y supervisión profesional de los RHS que se desempeñan en entornos rurales fue el más citado: catorce revisiones lo destacaron como uno de los factores que incide positivamente en la captación $(9,14,18)$, en la retención $(6,15,23-25)$ y en la captación o retención de RHS $(8,11,16,17)$. Asimismo, el aislamiento profesional y la insuficiente supervisión fueron señalados como factores que alejan a los trabajadores de los entornos RRD (16). Siete RS mencionaron las oportunidades de desarrollo de la carrera profesional como un factor que impacta en la captación o la retención $(8,10,11,12,16,17,19)$. Otro factor con impacto en la captación o retención es el grado de autonomía del trabajo en ámbitos rurales y remotos $(6,8,15,16)$, que puede constituir tanto un desafío y un elemento atractivo como una demanda que genera estrés e insatisfacción. También la Educación Médica Continua fue identificada como un factor que impacta en la retención de los RHS en ámbitos rurales o remotos $(6,10)$.

7) Factores asociados a las comunidades y estilo de vida rural: se asociaron con la retención de RHS en áreas rurales y fue jerarquizado en 9 de las 20 RS (6,14,15,16,19,21,23-25). Varios estudios enfatizaron que cuanto mayor sea la pertenencia e integración mayor probabilidad tendrán de permanecer en el medio.

\section{Evidencia sobre la efectividad de las intervenciones}

Se identificaron nueve revisiones: cinco RS $(22,27,28,29,30)$, una revisión realista (31) y tres revisiones de alcance $(3,32,33)$, dos de las cuales incluyen estudios multipaís $(3,32)$. La puntuación de AMSTAR de las RS varió en un rango de 5/11 $(29,30)$ a 10/11 (27). En la mayoría, la búsqueda fue exhaustiva; sin embargo, en ninguna fue posible realizar una síntesis cuantitativa de los resultados por la heterogeneidad de la evidencia identificada. La RS de la Cochrane, que limitó la selección de estudios a diseños randomizados o cuasi-randomizados con grupo control, identificó un único estudio. No se evaluó la calidad de los otros tipos de revisiones $(3,28,30)$.

Cinco RS evaluaron la efectividad de diferentes intervenciones $(22,27,28,31,32)$ y cuatro RS evaluaron una intervención en 
CUADRO 3. Principales características de los estudios que evaluaron captación o retención incluidos en las revisiones sobre efectividad de intervenciones

\begin{tabular}{|c|c|c|c|c|c|c|c|c|c|c|c|c|c|c|c|c|c|c|c|}
\hline \multirow[t]{2}{*}{ Autor } & \multirow[t]{2}{*}{ Tipo revisión } & \multirow[t]{2}{*}{ AMSTAR } & \multicolumn{5}{|c|}{ Población objetivo } & \multicolumn{3}{|c|}{ Áreas } & \multicolumn{4}{|c|}{ Tipo de Intervención } & \multirow[t]{2}{*}{ N estudios } & \multirow[t]{2}{*}{ País } & \multicolumn{2}{|c|}{ Desenlace } & \multirow{2}{*}{$\begin{array}{l}\text { Calidad } \\
\text { estudios }\end{array}$} \\
\hline & & & $\mathrm{Me}$ & Enf & Aux & TC & Otro & $\mathrm{Ru}$ & $\mathrm{Re}$ & $\overline{\mathrm{De}}$ & $\overline{E d}$ & Reg & Inc & SA & & & C & $\mathrm{R}$ & \\
\hline Hempe ${ }^{22}$ & RS & $8 / 11$ & & & & & & & & & & & & & 5 & EU & + & + & $\downarrow \downarrow$ \\
\hline Grobler ${ }^{27}$ & RS & $10 / 11$ & & & & & & & & & & & & & 1 & Tai & + & & $\downarrow$ \\
\hline \multirow[t]{8}{*}{ Puja Verma ${ }^{28}$} & \multirow[t]{8}{*}{ RS } & \multirow[t]{8}{*}{$7 / 11$} & & & & & & & & & & & & & 4 & Jap-Au-EU & + & + & $\downarrow \downarrow$ \\
\hline & & & & & & & & & & & & & & & 2 & EU & & $+/-$ & $\downarrow \downarrow$ \\
\hline & & & & & & & & & & & & & & & 2 & $\mathrm{NZ}$ & & - & $\downarrow \downarrow$ \\
\hline & & & & & & & & & & & & & & & 4 & Ru-EU-Au & & + & $\downarrow \downarrow$ \\
\hline & & & & & & & & & & & & & & & 7 & EU-Au-Ru & + & + & $\downarrow \downarrow$ \\
\hline & & & & & & & & & & & & & & & 2 & Au-Nor & & + & $\downarrow \downarrow$ \\
\hline & & & & & & & & & & & & & & & 4 & Nor-Ch-Ca & + & + & $\downarrow \downarrow$ \\
\hline & & & & & & & & & & & & & & & 1 & $\mathrm{Ca}$ & & - & $\downarrow \downarrow$ \\
\hline Ducat $^{29}$ & $\mathrm{RS}$ & $5 / 11$ & & & & & & & & & & & & & 1 & Aus & & + & $\downarrow \downarrow$ \\
\hline Bailey ${ }^{30}$ & $\mathrm{RS}$ & $5 / 11$ & & & & & & & & & & & & & 1 & Ma-Mo-Tan & & + & $\downarrow \downarrow$ \\
\hline Vasan $^{33}$ & RA & $\mathrm{N} / \mathrm{A}$ & & & & & & & & & & & & & 1 & Ma-Mo-Tan & & + & NR \\
\hline \multirow{6}{*}{ Vareilles $^{31}$} & \multirow{6}{*}{$\mathrm{RR}$} & \multirow{6}{*}{$\mathrm{N} / \mathrm{A}$} & & & & & & & & & & & & & 3 & EU-Is-Ke & + & + & NR \\
\hline & & & & & & & & & & & & & & & 1 & Gha & & + & NR \\
\hline & & & & & & & & & & & & & & & 1 & Ban & & + & NR \\
\hline & & & & & & & & & & & & & & & 1 & $\mathrm{Ug}$ & & + & NR \\
\hline & & & & & & & & & & & & & & & 1 & Cam & & + & NR \\
\hline & & & & & & & & & & & & & & & 4 & Ke-Ne-Ug-Sd & & + & NR \\
\hline \multirow[t]{3}{*}{ Krozen $^{32}$} & \multirow[t]{3}{*}{ RA-EM } & \multirow[t]{3}{*}{$N / A$} & & & & & & & & & & & & & 1 & Fra & + & + & NR \\
\hline & & & & & & & & & & & & & & & 4 & Bu-EE-Hu-Ru & $\mathrm{sd}$ & $\mathrm{sd}$ & NR \\
\hline & & & & & & & & & & & & & & & 1 & Fin & $\mathrm{sd}$ & $\mathrm{sd}$ & NR \\
\hline \multirow[t]{4}{*}{ Becerra $^{3}$} & \multirow[t]{4}{*}{ RA-EM } & \multirow[t]{4}{*}{$\mathrm{N} / \mathrm{A}$} & & & & & & & & & & & & & 1 & $\mathrm{Pe}$ & + & & \\
\hline & & & & & & & & & & & & & & & 2 & Bo-Ven & $\mathrm{sd}$ & $\mathrm{sd}$ & NR \\
\hline & & & & & & & & & & & & & & & 2 & Co-Ecu & $\mathrm{sd}$ & $\mathrm{sd}$ & NR \\
\hline & & & & & & & & & & & & & & & 1 & $\mathrm{Ch}$ & + & $\mathrm{sd}$ & NR \\
\hline
\end{tabular}

Fuente: elaboración propia.

RS: revisión sistemática; RA: revisión de alcance; EM: estudios multipaís; Me: medicina; Enf: enfermería; Aux: Disciplinas auxiliares; TC: Trabajador comunitario; Otro: otro personal; Ru: rural; Re: remota; Ed: educacional; Reg: regulatoria; Inc: incentivos; SA: Supervisión y apoyo; De: Desatendidas; EU: Estados Unidos; Tai: Taiwán; Jap: Japón; Au: Australia; NZ: Nueva Zelanda; Ru: Rumania; Nor: Noruega; Ch: Chile; Ca: Canadá; Ma: Malawi; Mo: Mozambique; Tan: Tanzania; Is: Israel; Ke: Kenia; Gha: Ghana; Ban: Bangladesh; Ug: Uganda; Cam: Camerún; Nep: Nepal; Sud: Sudáfrica; Fra: Francia; Hu: Hungría; Fin: Finlandia; Pe: Perú Bo: Bolivia; Ven: Venezuela; Co: Colombia; Ecu: Ecuador; s/d: sin datos; NR: No reportada; sd: sin datos

particular: tres RS evaluaron estrategias de supervisión/apoyo $(29,30,33)$ y una revisión de alcance seguida de un estudio de múltiples países en la Región de las Américas evaluó el impacto de programas de incentivos en áreas rurales o remotas de países en LAC (3).

En el cuadro 3 se describe el tipo y calidad de las revisiones seleccionadas, así como la población objetivo, área, tipo de intervención, resultados y calidad de los estudios que evaluaron la captación y retención de trabajadores/as incluidos en cada revisión.

\section{DISCUSIÓN}

Aunque existe un cuerpo de evidencia exhaustivo sobre los factores que inciden en la captación y retención de RHS, la calidad de los estudios incluidos fue limitada. El número de revisiones sobre efectividad de intervenciones fue menor, aunque con iguales limitaciones en la calidad metodológica.

\section{Captación y retención: distintos desenlaces, distintos factores}

Uno de los hallazgos más significativos de esta revisión se asoció con las diferencias en los patrones de factores que inciden en la captación y en la retención de los trabajadores/as en las áreas rurales y remotas.

En el caso de la captación, el origen rural y experiencias satisfactorias de exposición a dichos ámbitos durante la formación de grado o la residencia en los primeros años después de graduarse favorece la captación de trabajadores en áreas RRD (7-9,13,14,1719,21-24,26). Sin embargo, sólo se mantendrá la decisión de permanecer en la medida en la que las CyMAT, estén acordes a sus necesidades y demandas, y favorezcan su permanencia en estas áreas $(6,10,14,15,16,19-21,22-25)$. Dichas necesidades y demandas son dinámicas y están afectadas tanto por factores internos -propios del trabajador como el ciclo de vida personal, familiar y laboral- y por factores externos, como cambios en la comunidad o reformas de salud $(6,34)$. De acuerdo con lo observado, el rol social de las universidades debería ser siempre considerado entre los factores que inciden en la captación y retención.

La evidencia identificada muestra que los factores individuales, y en particular, el origen y la exposición al medio rural impactan positivamente en las expectativas del trabajador. La posibilidad de trabajar en red o recibir supervisión o apoyo profesional incidiría tanto en la captación como en la retención de los trabajadores de salud, y esto estaría reafirmando lo que destaca Campbell en cuanto al efecto expulsivo que tiene el aislamiento sobre los RHS en estas áreas (16). 
En el caso de la retención se trata de un balance entre las necesidades y demandas reales del trabajador; aquí todos los elementos del modelo conceptual propuesto sobre CyMAT cobran preponderancia.

\section{Efectividad de las intervenciones: escaso progreso en la producción de evidencia}

La evidencia de alta calidad sobre la efectividad de las intervenciones dirigidas específicamente a las CyMAT para mejorar la atracción, captación y retención de los RHS en áreas RRD es aún muy limitada. En el año 2010, la OMS señaló la necesidad de promover estudios de mayor calidad metodológica (4) y los progresos en este sentido han sido muy escasos.

La dificultad para producir este tipo de evidencia puede explicarse, en parte, por la naturaleza de las intervenciones que se evalúan, que suelen ser multifacéticas, y por tanto, complejas. Tanto su diseño como su implementación son altamente dependientes del contexto $(4,32,35)$, lo cual puede explicar también la variabilidad observada con relación a la efectividad de un mismo tipo de intervención. Esta revisión identificó una multiplicidad de factores a nivel micro, meso y macro, que inciden en la captación o en la retención de los trabajadores de salud en áreas RRD. La preponderancia de cada uno de estos factores depende de cada contexto y del desenlace de interés. Por tanto, el diseño de este tipo de intervenciones debería apoyarse en un adecuado diagnóstico de situación, que identifique el tipo de desenlace, los factores determinantes, el grupo profesional objetivo y las áreas geográficas involucradas, ya que se pueden requerir enfoques diferentes en cada caso.

Por otra parte, la evaluación de este tipo de intervenciones requiere un enfoque teórico-metodológico diferente, que considere no sólo la evaluación de la efectividad, sino también de las estrategias de implementación que se emplean. Este tipo de diseños suele demandar recursos financieros y humanos calificados no siempre disponibles, sobre todo en países de bajos y medianos ingresos.

El uso de incentivos fue el tipo de intervención más frecuentemente considerada en los distintos estudios identificados. En general, se observó que esta intervención tuvo un efecto positivo en la captación o en la retención de trabajadores en áreas RRD. Sin embargo, la calidad de los estudios que se incluyeron es muy limitada y la modalidad en la que se los empleó fue diferente (aislados, combinados con otra intervención o incluidos en un paquete de intervenciones). La única revisión realizada en LAC evaluó el uso de incentivos financieros y no-financieros implementados en nueve países de la Región de las Américas (3), mediante doce programas de los cuales solo dos registraron datos oficiales de impacto.

La principal limitación del estudio se relaciona con la naturaleza de su diseño, dado que se trata de una revisión de revisiones denominada revisión rápida. La misma se realiza en un tiempo acotado no mayor a tres meses, lo que permite adecuaciones metodológicas que pueden resultar en sesgos que limitan la generalización de sus resultados. Aunque la búsqueda incluyó áreas desatendidas, casi la totalidad de los resultados correspondieron a áreas remotas y rurales. Se excluyeron estudios sobre intervenciones dirigidas a estudiantes. A pesar de estas limitaciones, sus resultados y su integración con un modelo teórico-conceptual sobre las CyMAT permitieron actualizar y complementar la evidencia difundida por la OMS en 2010 (4).

En conclusión, la evidencia sobre factores que inciden en las CyMAT y se relacionan positivamente con la captación y retención de trabajadores en el primer nivel de atención de áreas RRD es suficiente y debería ser tenida en cuenta al diseñar intervenciones. En contraste, la evidencia de alta calidad sobre la efectividad de intervenciones es aún escasa y denota la necesidad de promover estudios controlados, con rigurosidad metodológica, en particular que sean representativos de experiencias en la Región de las Américas.

Los diseños no controlados u observacionales no serían tan necesarios por cuanto no contribuirían a ampliar el conocimiento ya disponible sobre esta temática y representarían una inversión ineficiente en investigación.

Contribuciones de los autores. ZO, MEE, CM y LA concibieron el estudio original; $\mathrm{MEE}, \mathrm{ZO}$ y LA recolectaron, analizaron, interpretaron los resultados y escribieron el manuscrito. Todos los autores revisaron y aprobaron la versión final.

Conflictos de intereses. Ninguno declarado.

Declaración. Las opiniones expresadas en este manuscrito son únicamente responsabilidad de los autores y no reflejan necesariamente los de la Revista Panamericana de Salud Pública o la Organización Panamericana de la Salud.

\section{REFERENCIAS}

1. Organización Panamericana de la Salud. $53^{\circ}$ Consejo Directivo de la OPS, $66^{\text {a }}$ sesión del Comité Regional de la OMS para las Américas. Estrategia para el acceso universal a la salud y la cobertura universal de salud. Documento CD53/5, Rev. 2 de octubre del 2014. Disponible en: https://www.paho.org/hq/dmdocuments/2014/ CD53-5-s.pdf

2. Dal Poz M R, Sepulveda H R, Costa Couto MH, Godue C, Padilla M, Cameron R et al. Assessment of human resources for health programme implementation in 15 Latin American and Caribbean countries. Hum Resour Health 2015;13:24-32. https://doi.org/ 10.1186/s12960-015-0016-4

3. Becerra C; Cañizares Fuentes R; Bustos V. Desarrollo de Esquemas de Incentivos para la Retención - Fidelización del Personal de Salud en Áreas Rurales y de Difícil Acceso. En Cañizares Fuentes R, García Gutiérrez JF (editores). Planificación y gestión de recursos humanos en salud en los países andinos. Evidencia para la toma de decisiones. Organismo Andino de Salud-Convenio Hipólito Unanue, Lima, 2015. p. 361-405.

4. Dolea C, Stormont L, Braichet JM. Evaluated strategies to increase attraction and retention of health workers in remote and rural areas. Bull World Health Organ. 2010;88(5):379-385. doi: 10.2471/ BLT.09.070607

5. Antonietti L, Ortiz Z, Esandi ME, Duré I, Cho M. Condiciones y medio ambiente de trabajo en salud: modelo conceptual para áreas remotas y rurales. Rev Panam Salud Publica. 2020;44:e111. https:/ / doi.org/10.26633/RPSP.20120.111

6. Humphreys J, Jones J, Jones M, Bambford E, Taylor D. A critical review of rural medical workforce retention in Australia. Aust Health Rev. 2001; 24(4):91-102. https://doi.org/10.1071/ AH010091a

7. Brooks RG, Walsh M, Mardon RE, Lewis M, Clawson A. The roles of nature and nurture in the recruitment and retention of primary care 
physicians in rural areas: a review of the literature. Acad Med. 2002; 77(8):790-8. https://doi.org/10.1097/00001888-200208000-00008

8. Bilodeau H, Leduc N. Inventory of the main factors determining the attraction, installation and retention of physicians in remote areas. Cah Sociol Demogr Med. 2003;43(3):485-504.

9. Philipp D, Wright D. Recruiting Healthcare Professionals to Rural Areas. Radiol Manag. 2005:27(6):44-50.

10. Glazebrook RM, Harrison SL. Obstacles and solutions to maintenance of advanced procedural skills for rural and remote medical practitioners in Australia. Rural Remote Health. 2006;6 (4):502.

11. Lehmann U, Dieleman M, Martineau T. Staffing remote rural areas in middle- and low-income countries: a literature review of attraction and retention. BMC Health Serv Res. 2008;8:19. https://doi. org /10.1186/1472-6963-8-19

12. Willis-Shattuck M, Bidwell P, Thomas S, Wyness L, Blaauw D, Ditlopo P. Motivation and retention of health workers in developing countries: a systematic review. BMC Health Serv Res. 2008;8:247. https:/ / doi.org/10.1186/1472-6963-8-247

13. Ballance D, Kornegay D, Evans P. Factors that influence physicians to practice in rural locations: a review and commentary. J Rural Health. 2009; 25(3):276-81. https://doi.org/10.1111/j. 1748-0361.2009.00230.x

14. Henry JA, Edwards BJ, Crotty B. Why do medical graduates choose rural careers? Rural Remote Health. 2009;9(1):1083.

15. Roberge C. Who stays in rural nursing practice? An international review of the literature on factors influencing rural nurse retention. Online J Rural Nurs Health Care. 2009; 9. https: / / doi.org/10.14574/ ojrnhc.v9i1.107

16. Campbell N, McAllister L, Eley D. The influence of motivation in recruitment and retention of rural and remote allied health professionals: a literature review. Rural Remote Health. 2012; 12:1900.

17. Roots $\mathrm{R}, \mathrm{Li} \mathrm{L}$. Recruitment and retention of occupational therapists and physiotherapists in rural regions: A meta-synthesis. BMC Health Serv Health Serv Res. 2013;13:59. https://doi.org/10.1186/ 1472-6963-13-59

18. Trepanier A, Gagnon MP, Mbemba GI, Coté J, Paré G, Fortin JP et al. Factors associated with intended and effective settlement of nursing students and newly graduated nurses in a rural setting after graduation: a mixed-methods review. Int J Nurs Stud. 2013; 50 (3):314-25. https://doi.org/10.1016/j.ijnurstu.2012.09.005

19. Viscomi M, Larkins S, Gupta TS. Recruitment and retention of general practitioners in rural Canada and Australia: a review of the literature. Can J Rural Med. 2013; 18 (1):13-23.

20. Mbemba GI, Gagnon MP, Hamelin-Brabant L. Factors Influencing Recruitment and Retention of Healthcare Workers in Rural and Remote Areas in Developed and Developing Countries: An Overview. J Public Health Afr. 2016;7(2):565. https://doi.org/10.4081/ jphia.2016.565

21. Godwin DM, Hoang H, Crocombe LA, Bell E. Dental practitioner rural work movements: a systematic review. Rural Remote Health. 2014;14(3):2825.

22. Hempel S, Gibbons MM, Ulloa JG, Macqueen IT, Miake-Lye IM, Beroes JM, et al. Rural Healthcare Workforce: A Systematic Review. Washington (DC): Department of Veterans Affairs (US); 2015

23. Goodfellow A, Ulloa JG, Dowling PT, Talamantes E, Chheda S, Bone C, et al. Predictors of Primary Care Physician Practice Location in Underserved Urban or Rural Areas in the United States: A Systematic Literature Review. Acad Med. 2016; 91(9):1313-21. https://doi. org/10.1097/ACM.0000000000001203
24. Parlier AB, Galvin SL, Thach S, Kruidenier D, Fagan EB. The Road to Rural Primary Care: A Narrative Review of Factors That Help Develop, Recruit, and Retain Rural Primary Care Physicians. Acad Med. 2018; 93(1):130-140. https://doi.org/10.1097/ acm.0000000000001839

25. Russell DJ, McGrail MR, Humphreys JS. Determinants of rural Australian primary health care worker retention: A synthesis of key evidence and implications for policymaking. Aust J Rural Health. 2017; 25(1):5-14. https://doi.org/10.1111/ajr.12294

26. Fleming P, Sinnot ML. Rural physician supply and retention: factors in the Canadian context. Can J Rural Med. 2018; 23(1):15-20.

27. Grobler L, Marais BJ, Mabunda S. Interventions for increasing the proportion of health professionals practising in rural and other underserved areas. Cochrane Database Syst Rev. 2009; 21(1):CD005314. https://doi.org/10.1002/14651858.CD005314.pub2

28. Verma P, Ford J, Stuart A, Howe A, Everington S, Steel N. A systematic review of strategies to recruit and retain primary care doctors. BMC Health Serv Res. 2016;16:126. https://doi.org/10.1186/ s12913-016-1370-1

29. Ducat WH, Kumar S. A systematic review of professional supervision experiences and effects for allied health practitioners working in non-metropolitan health care settings. J Multidiscip Healthc. 2015;26(8):397-407. https://doi.org/10.2147/JMDH.S84557

30. Bayley C, Blake C, Schriver M, Kalumire C, Thomas T, Hilber A. A systematic review of supportive supervision as a strategy to improve primary healthcare services in Sub-Saharan Africa. Int J Gynaecol Obstet. 2016;132(1):117-25. https://doi.org/10.1016/j.ijgo.2015.10.004

31. Vareilles G, Pommier J, Marchal B, Kane S. Understanding the performance of community health volunteers involved in the delivery of health programmes in underserved areas: a realist synthesis. Implement Sci. 2017;12(1):22. https://doi.org/10.1186/ s13012-017-0554-3

32. Kroezen M, Dussault G, Craveiro I, Dieleman M, Jansen C, Buchan J. et al. Recruitment and retention of health professionals across Europe: A literature review and multiple case study research. Health Policy. 2015;119 (12):1517-28. https://doi.org/10.1016/j. healthpol.2015.08.003

33. Vasan A, Mabey DC, Chaudhri S, Brown Epstein HA, Lawn SD. Support and performance improvement for primary health care workers in low- and middle-income countries: a scoping review of intervention design and methods. Health Policy Plan. 2017;32(3):437-452. https://doi.org/10.1093/heapol/czw144

34. Cosgrave C; Malatzky C; Gillespie J. Social Determinants of Rural Health Workforce Retention: A Scoping Review. Int J Environ Res Public Health. 2019;16 (3):314. https://doi.org/10.3390/ijerph 16030314

35. Liu X, Dou L, Zhang H, Sun Y, Yuan B. Analysis of context factors in compulsory and incentive strategies for improving attraction and retention of health workers in rural and remote areas: a systematic review. Human Resour Health. 2015;13:61. https://doi. org/10.1186/s12960-015-0059-6

Manuscrito recibido el 13 de enero de 2020. Aceptado para su publicación, tras revisión, el 29 de junio de 2020. 


\section{Factors and interventions that affect working conditions and environment to increase the attraction, recruitment and retention of human resources for health at the primary care level in rural, remote or underserved areas}

ABSTRACT Objective. To identify and systematize available empirical evidence on factors and interventions that affect working conditions and environment in order to increase the attraction, recruitment and retention of human resources for health at the primary care level in rural, remote or underserved areas.

Methods. Rapid review of reviews selected according to relevance, eligibility and inclusion criteria. The search was conducted on electronic and manual databases, including grey literature. AMSTAR I was used to assess the quality of systematic reviews and a thematic analysis for synthesis of the results.

Results. Sixteen reviews were included, one of which contained 14 reviews. Of the total, 20 reviews analyzed factors and 9 evaluated the effectiveness of interventions. The evidence on factors is abundant, but of limited quality. Individual, family and "previous exposure to a rural setting" factors were associated with higher recruitment; organizational and external context factors were important for human resource retention. Networking and professional support influenced recruitment and retention. Evidence on the effectiveness of interventions was limited, both in quantity and quality. The most frequently used intervention was incentives.

Conclusions. Evidence on factors that are positively related to recruitment and retention of workers at the first level of care in rural, remote or underserved areas is sufficient and should be taken into account when designing interventions. Quality evidence on the effectiveness of interventions is scarce. More controlled studies with methodological rigor are needed, particularly in the Americas.

Keywords

Working conditions; rural areas; remote areas; medically underserved areas; workforce; primary health care; personnel selection. 